\title{
Real-world evaluation of a computed tomography- first triage strategy for suspected coronavirus disease 2019 in emergency outpatients in Japan: an observational cohort study
}

\section{Shigeta Miyake}

Yokohama City University

Takuma Higurashi ( $\nabla$ takuma_h@yokohama-cu.ac.jp )

Yokohama City University

Takashi Jono

Yokohama City University

Taisuke Akimoto

Yokohama City University

Fumihiro Ogawa

Yokohama City University

Yasufumi Oi

Yokohama City University

Katsushi Tanaka

Yokohama City University

Yu Hara

Yokohama City University

Nobuaki Kobayashi

Yokohama City University

Hideaki Kato

Yokohama City University

Tsuneo Yamashiro

Yokohama City University

Daisuke Utsunomiya

Yokohama City University

Atsushi Nakajima

Yokohama City University

Tetsuya Yamamoto

Yokohama City University

Shin Maeda

Yokohama City University 


\section{Takeshi Kaneko}

Yokohama City University

Ichiro Takeuchi

Yokohama City University

\section{Research Article}

Keywords: Computed tomography, Coronavirus disease 2019, Diagnosis, Emergency treatment, Fever, Pneumonia, computed tomography-first triage

Posted Date: October 27th, 2020

DOl: https://doi.org/10.21203/rs.3.rs-36430/v2

License: (c) (i) This work is licensed under a Creative Commons Attribution 4.0 International License. Read Full License

Version of Record: A version of this preprint was published at Medicine on June 4th, 2021. See the published version at https://doi.org/10.1097/MD.0000000000026161. 


\section{Abstract}

Background: Coronavirus disease 2019 (COVID-19) continues to spread worldwide. Because of the absence of reliable rapid diagnostic systems, patients with COVID-19 symptoms are suspected of disease. Computed tomography (CT) in patients with suspected COVID-19 may be reasonable for triaging, and CT-first triage strategies have been proposed. However, clinical evaluation of a CT-first triage protocol is lacking. The aim of this study is to investigate the real-world efficacy and limitations of a CTfirst triage strategy in patients with suspected COVID-19.

Methods: This was a single-center cohort study evaluating outpatients with suspected COVID-19 who underwent a medical examination at Yokohama City University Hospital and who were prospectively registered between 9 February and 5 May 2020. We treated patients according to the CT-first triage protocol. CT findings were classified into five categories according to the COVID-19 Reporting and Data System (CO-RADS). With the CT-first triage protocol, patients with a suspicious clinical history, symptoms, or suspicious findings on chest CT were allocated to the COVID-19 suspected group. The primary outcome was efficacy of the CT-first triage protocol for outpatients with suspected COVID-19. We conducted additional analyses of the isolation time of outpatients with suspected COVID-19 and reached final diagnoses.

Results: In total, 108 outpatients with suspected COVID-19 were examined at our hospital. Forty-eight patients (44.9\%) were categorized as CO-RADS 1, 26 patients (24.3\%) as CO-RADS 2, 14 patients (13.1\%) as CO-RADS 3,6 patients (5.6\%) as CO-RADS 4, and 13 patients (12.1\%) as CO-RADS 5. One patient was excluded because of pregnancy. Using the CT-first triage protocol, 48 (44.9\%) patients were suspected of having COVID-19. Nine patients (18.8\%) in this group were positive for severe acute respiratory syndrome coronavirus 2 using polymerase chain reaction; no patients in the group not suspected of having COVID19 were diagnosed with COVID-19 during follow up. The protocol significantly shortened the duration of isolation for the not-suspected versus the suspected group (70.5 vs. 1037.0 minutes, $P<.001$ ).

Conclusions: Our CT-first triage protocol was acceptable for triaging outpatients with suspected COVID19. This protocol will be helpful for appropriate triage, especially in areas where polymerase chain reaction is limited.

\section{Introduction}

The pandemic of coronavirus disease 2019 (COVID-19) is currently one of the greatest human challenges for health care systems and economic systems worldwide. ${ }^{1-3}$ COVID-19, caused by severe acute respiratory syndrome coronavirus 2 (SARS-CoV-2), has affected more than 4.6 million individuals, and has caused over 300,000 deaths to date. ${ }^{4}$ The severity and case fatality rate of COVID-19 differs from country to country, depending on the health care system and health policies. ${ }^{5,6}$ Generally, the excess burden surpasses healthcare capacity for patients in intensive care and causes medical collapse, which may trigger an increase in the death rate. 
Several studies have revealed factors associated with poor prognosis such as older age, underlying comorbidities, clinical symptoms, and laboratory findings. ${ }^{7}$ Because hospitalized patients constitute a high-risk population, hospital outbreaks are among the most dangerous situations. Therefore, adequate use of disposable personal protective equipment (PPE) is recommended. ${ }^{8}$ Because of a global shortage of PPE, there are many reports of hospital outbreaks of COVID-19 and infection of medical staff. To prevent such outbreaks in hospitals, it is advisable to separate services for patients with suspected COVID-19. ${ }^{5}$

To reduce consumption of PPE and implement appropriate isolation, triage to distinguish patients who are less likely to have COVID-19 from those with suspected COVID-19 is necessary. ${ }^{9,10}$ However, there are no established protocols for managing patients with suspected COVID-19 who present to the emergency department. Because reliable rapid diagnostic systems are lacking, patients with symptoms related to COVID-19 such as fever, fatigue, respiratory symptoms, headache, and taste or olfactory disorders are widely treated as suspected COVID-19. Several triage protocols and their efficiency have been reported. ${ }^{9,11,12}$ However, because COVID-19 symptoms are not specific to that disease, there might be many differential diagnoses in patients with suspected COVID-19. Recently, radiological findings of COVID-19 have been reported. ${ }^{12-14}$ Generally, chest X-ray is useful to diagnose pneumonia; however, it is unsuitable for COVID-19. Because the characteristics of chest radiological images in COVID-19 mainly appear in the interstitium, revealing ground-glass opacities (GGO), it is difficult to diagnose COVID-19 using chest X-ray alone, especially in mild cases. Presently, computed tomography (CT) in patients with COVID-19 can be considered a reasonable method for triaging patients with COVID-19. However, consensus has not been reached regarding clinical evaluation of triage protocols using $\mathrm{CT} .^{15}$

COVID-19 has affected 17,018 individuals and caused 903 deaths in Japan as of 3 June, 2020. ${ }^{16}$ Yokohama City is the second largest city in Japan. Since February 2020, we have treated patients with COVID-19 at Yokohama City University Hospital, a tertiary-level hospital, owing to the outbreak on the "Diamond Princess" cruise ship docked in Yokohama Bay. ${ }^{17,18}$ A select team (Team COVID-19) was deployed for the treatment of patients with moderate to severe COVID-19 and with suspected COVID-19. One focus of Team COVID-19 is on preventing in-hospital infections and medical staff infections, with reference to protocols that were previously launched during the epidemic of a novel type of influenza in 2002 to 2003. Team COVID-19 comprises 10 doctors (mainly from the internal medicine and surgery departments) and engages with the outpatient clinic and hospitalized patients with suspected COVID-19 in a 24-hour rotation schedule. At our hospital, a protocol adopting CT as the first-line examination, the "CT-first triage protocol," has been used in the management of patients with suspected COVID-19. We adopted the CT-first triage protocol for several reasons. There are not many patients with COVID-19 in Japan; thus, polymerase chain reaction (PCR) testing of individuals with no or mild symptoms might lead to many false-negative results because of low sensitivity of the PCR test. ${ }^{19,20}$ Furthermore, in some regions, such as Wuhan and Italy, many people with mild symptoms rushed to a hospital to receive PCR testing, leading to the collapse of medical services and subsequently, many in-hospital infections. To avoid such events and keep hospitals functioning normally, adopting the CT-first triage protocol was 
reasonable in Japan. To verify the efficacy and limitations of this protocol, we focused on the characteristics of outpatients with suspected COVID-19 using the CT-first triage protocol. To our knowledge, this is the first detailed report using real-world clinical data regarding a CT-first triage protocol used for patients with suspected COVID-19.

\section{Methods}

\section{Study design and setting}

This was a single-center cohort study aiming to evaluate outpatients with suspected COVID-19 who received medical examination at Yokohama City University Hospital, prospectively registered between 9 February, 2020 and 5 May, 2020.

\section{Ethical approval}

The Institutional Review Board at Yokohama City University Hospital approved this study (approval number B200200047). Consent for participation in this study was obtained from all patients after explaining the clinical study by verbal or a description of how to opt out. (https://www.yokohamacu.ac.jp/amedrc/ethics/ethical/fuzoku_optout.html).

\section{Participants}

Among all patients who visited our outpatient clinic, those who exhibited COVID-19-like symptoms such as fever (over $37.5^{\circ} \mathrm{C}$ ), fatigue, respiratory symptoms, headache, and taste or olfactory disorders, were identified and instructed to visit our separate clinic as outpatients with suspected COVID-19.

We analyzed the following baseline patient characteristics: age, sex, history of overseas travel, contact history with a confirmed COVID-19 case, and underlying comorbidities (chronic pulmonary disease, diabetes, hypertension, chronic renal failure, cardiovascular disease, cerebrovascular disease, and malignancy). We also evaluated patients' existing symptoms such as fever, fatigue, respiratory symptoms, headache, and taste or olfactory disorders.

\section{Interventions and measurements}

\section{CT-first triage protocol}

First, patients complaining of fever, fatigue, respiratory symptoms, headache, and taste or olfactory disorder were redirected to the separate suspected COVID-19 outpatient clinic of the emergency room described above. From that time, patients were isolated from other patients and treated as if they had COVID-19. Second, a nurse in the outpatient clinic equipped with PPE briefly checked patients' vital signs and their present histories. Then, chest CT was conducted. Our hospital was equipped with three CT scanning suites. We set one of these as a dedicated suite for patients with suspected or confirmed COVID-19. Patients with suspected COVID-19 were separated from other patients in the transport 
corridors and elevators to the CT suite. An 80-row CT scanner (Aquilion Prime, Canon Medical Systems, Otawara, Tochigi, Japan) was used in this study. The tube voltage setting was $120 \mathrm{kVp}$ and automatic exposure control was applied. The average CT dose index volume (CTDIvol) was $9.4 \pm 4.1 \mathrm{mGy}$. These settings were not specialized for CT screening of COVID-19 pneumonia, and a reduction in radiation exposure was not applied. Patients were scanned during full inspiratory breath hold; however, some patients with severe dyspnea were scanned without performing a breath hold. Two-pattern CT images were reconstructed with a slice thickness/increment of $5.0 \mathrm{~mm} / 5.0 \mathrm{~mm}$ and $0.5 \mathrm{~mm} / 0.5 \mathrm{~mm}$. Coronal and sagittal multiplanar reconstruction images were also acquired. All CT scans were immediately interpreted by trained radiologists. During regular business hours, all CT scans were interpreted and full reports were created by board-certified radiologists from the Japan Radiological Society (10 radiologists with 6-26 years' experience in chest radiology). Scans were also immediately checked, and necessary information on the possibility of COVID-19 pneumonia was reported to COVID-19 doctors after hours by the 10 boardcertified radiologists and 9 fellows from the Department of Radiology. If necessary, fellows contacted board-certified radiologists for their opinions. CT findings were classified into five categories according to the COVID-19 Reporting and Data System (CO-RADS). ${ }^{21}$ The CO-RADS was used to assess suspected pulmonary involvement in COVID-19 on a scale from 1 (very low) to 5 (very high), as follows: CO-RADS 1 , normal CT or non-infectious etiology (e.g., lung tumor, lung fibrosis); CO-RADS 2, infectious etiology that is not compatible with COVID-19 (e.g., infectious bronchiolitis, bronchopneumonia, lobar pneumonia); CORADS 3, equivocal findings for COVID-19 (e.g., homogeneous extensive GGO); CO-RADS 4, typical CT findings of COVID-19 (similar to CO-RADS 5) but showing some overlap with other pneumonia; CO-RADS 5, typical CT findings of COVID-19 (e.g., multifocal GGO with or without consolidation in lung regions close to visceral pleural surfaces). Representative images in each category are shown in Figure 1. Finally, patients were allocated to a "COVID-19 suspected" group or a "COVID-19 less likely" group. Patients with a suspicious clinical history (contact with an infected person and overseas travel history), suspicious findings on chest CT scan (CO-RADS 3 to 5), or who were suspected of having COVID-19 according to their clinical symptoms that were difficult to explain by the underlying disease were included in the COVID-19 suspected group. Patients with neither a suspicious clinical history nor chest CT features suggestive of COVID-19 (CO-RADS 1 to 2) were included in the COVID-19 less likely group. If patients were diagnosed as less likely to have COVID-19, isolation and PPE support were discontinued, and detailed physical and laboratory examinations were performed. However, if patients were diagnosed with COVID19 suspected, isolation and PPE support were continued until a negative PCR test result was obtained for the patient. A PCR test for SARS-CoV-2 was performed only in COVID-19 suspected group patients. All samples obtained after 11:00 on the previous day were immediately submitted for PCR testing each day, and the results were obtained at 15:00 on the same day.

\section{Outcomes}

The primary outcome was the efficacy of the CT-first triage protocol in outpatients with suspected COVID19. We assessed all results of PCR for SARS-CoV-2 and the final diagnoses of patients. Patients who did not undergo PCR testing were followed for up to 14 days until confirmed clinically negative for COVID-19. 
Secondary outcomes were the duration of isolation among all patients and safety of the medical staff and hospitalized patients. Isolation duration was defined as the time that PPE was required or that patients were isolated from medical staff and other patients to prevent COVID-19 infection. For the COVID-19 suspected group, the time to a PCR-confirmed negative result was applicable for determining the duration of isolation. The isolation duration of PCR-positive patients was excluded from the results for isolation duration in the COVID-19 suspected group because COVID-19-positive patients remained in isolation until their treatment was finished. However, in the COVID-19 less likely group, the time to a CTconfirmed negative result was applicable to determine the isolation duration. The patient data were retrospectively examined using medical records.

\section{Statistical analysis}

To account for biases, we performed the analysis after the study period was complete. Results are presented as mean \pm standard deviation for quantitative data and as frequency (percentage) for categorical data. The Student's $t$-test was used for continuous data. A $P$ value $<.05$ was considered statistically significant. All statistical analyses were performed using Prism $7.9 \mathrm{~J}$ for Windows (GraphPad Software, Inc., San Diego, CA, USA).

\section{Results}

\section{Characteristics of outpatients with suspected COVID-19}

Between 9 February, 2020 and 5 May, 2020, a total 108 outpatients with suspected COVID-19 underwent a medical examination at our hospital, and all of them were included in this study. The mean participant age was $58.9 \pm 19.5$ years (range, 18 to 101 years), and 60 patients (55.6\%) were male. Four patients had an overseas travel history and eight patients had contact with a confirmed COVID-19 case. Nearly $70 \%$ of patients had comorbidities such as chronic pulmonary disease $(21,19.4 \%)$, hypertension $(25,23.1 \%)$, and malignancy $(20,18.5 \%)$. The most common symptom was fever $(77,71.2 \%)$ and 55 patients $(50.9 \%)$ reported respiratory symptoms (Table 1 ).

\section{Triage results of the CT-first triage protocol}

In conducting the CT-first triage protocol, one patient refused chest CT because she was pregnant. The remaining outpatients with suspected COVID-19 in the clinic received chest CT and all images were immediately categorized according to the CO-RADS by specialized radiologists and Team COVID-19 doctors. Forty-eight (44.9\%) patients were categorized as CO-RADS 1, 26 (24.3\%) CO-RADS 2, 14 (13.1\%) CO-RADS 3, 6 (5.6\%) CO-RADS 4, and $13(12.1 \%)$ patients were categorized as CO-RADS 5. Finally, 48 $(44.9 \%)$ patients were included in the COVID-19 suspected group. On chest CT, two-thirds of patients in the suspected COVID-19 group showed features of CO-RADS categories 3 to 5 ; the remaining patients in the suspected COVID-19 group were classified according to their clinical history and other examinations conducted by Team COVID-19 doctors. All patients in the COVID-19 less likely group showed features of 
CO-RADS categories 1 or 2 and had no clinical history suspicious for COVID-19. The participant flow is shown in Figure 2.

Isolation was continued until patients were diagnosed as either unlikely to have COVID-19 using the CTfirst triage protocol or had a confirmed negative PCR test result for SARS-CoV-2. The mean duration of isolation was significantly shorter in the COVID-19 less likely group (70.5 \pm 41.2 minutes) than in the COVID-19 suspected group (1037.0 \pm 512 minutes) $(P<0.001)$ (Table 2$)$.

\section{Final clinical diagnosis after CT-first triage protocol}

All patients in the COVID-19 suspected group underwent PCR testing for SARS-Co-V-2. Among 48 patients in this group, 9 (18.8\%) were positive for SARS-CoV-2 on PCR and the remainder were negative. One patients with suspected COVID-19 had one negative PCR test result; however, Team COVID-19 doctors strongly suspected COVID-19 because the patient was categorized as CO-RADS 5 according to CT findings; the patient was retested and definitively diagnosed with COVID-19 after two positive PCR test results. The others eight of nine patients definitely diagnosed with COVID-19 had CT features of CO-RADS category 5 . However, during the study period, no patients were subsequently diagnosed with COVID-19 in the group less likely to have COVID-19. The final diagnoses of outpatients with suspected COVID-19 who did not have COVID-19 were respiratory infection $(51,47.7 \%)$, other focal infection $(22,20.6 \%)$, tumorrelated fever $(5,4.7 \%)$, and interstitial pneumonia (4, 3.7\%). Seven outpatients (6.5\%) had life-threatening conditions that mimicked COVID-19: four with meningitis, one with acute myocardial infarction, and one patient who had pneumonia with venous thrombosis. Cases of life-threating conditions among outpatients with suspected COVID-19 are described in the Supplemental material. A total 31 patients $(64.6 \%)$ in the COVID-19 suspected group and 20 patients (33.9\%) in the COVID-19 less likely group were hospitalized. One patient with suspected COVID-19 died owing to severe COVID-19 causing multiple organ failure, and one patient in the COVID-19 less likely group died owing to acute myocardial infarction (Table 3).

\section{Infection control during the study period}

Via separation of outpatients with suspected COVID-19 in our specialized outpatient clinic and using the CT-first triage protocol, adequate isolation was achieved for these outpatients. Throughout the study period, no medical staff developed fever or other health concerns. In addition, no in-hospital infections occurred, and we were able to use less PPE.

\section{Discussion}

In this study, we report the evaluation results for the CT-first triage protocol in outpatients with suspected COVID-19 at our clinic. To our knowledge, these are the first real-world clinical data regarding a CT-first triage protocol worldwide. In our experience, most outpatients with suspected COVID-19 did not have COVID-19 (Table 1). From the perspective of conserving medical resources, a reasonable triage protocol is essential. We demonstrated the effectiveness of our CT-first triage protocol in selected outpatients 
suspected of having COVID-19 based on their clinical history and chest CT features. In our experience, the CT-first triage protocol is acceptable for clinical use. Our CT-first triage protocol did not miss any patients who were ultimately confirmed to have COVID-19 and improved the pre-test probability of PCR testing for SARS-CoV-2 from $8.4 \%$ to $18.8 \%$ (Table 2). Furthermore, no medical staff or hospitalized patients developed new COVID-19-related symptoms during the study period. The consumption of PPE was also kept to a minimum.

To respond to the threat of infectious diseases, reliable rapid diagnostic technologies like that for influenza are required. The use of new technologies including artificial intelligence $(\mathrm{Al})^{22}$, specific antibody ${ }^{23}$, and immunochromatography ${ }^{24}$ has recently been reported. For clinical use, these new technologies clearly require additional studies and concrete evidence. Nevertheless, increasing pre-test probability using Al-based medical interviews and Al analysis of CT scans are promising directions. ${ }^{25}$ Our hospital is equipped with three CT scanning suites and one is a dedicated suite for patients with suspected or confirmed COVID-19. Therefore, the CT-first triage protocol is currently feasible for screening suspected cases of COVID-19. Whereas the CO-RADS categories are reliable, owing to the existence of false-negative CT categorization, ${ }^{21}$ a protocol combining the clinical history of COVID-19 risk factors and CT features is recommended. Prokop et al. reported that $13.8 \%$ (95\% confidence interval $9 \%-18 \%$ ) of cases were false negatives in their cohort of 840 images. ${ }^{21}$ We using CO-RADS and patients' clinical histories for diagnosis; there were no patients diagnosed with COVID-19 in the group classified as less likely to have COVID-19 during the follow-up period.

One of the merits of our CT-first triage protocol is the ability to quickly release patients from isolation who are unlikely to have COVID-19 (Table 2). In general, COVID-19 was diagnosed with PCR testing for SARSCoV-2. However, PCR tests normally require at least several hours, and the PCR test for SARS-CoV-2 diagnosis takes longer because it is performed using several specimens at once. In that sense, the CT-first protocol is an acceptable method to judge whether patients who are unlikely to have COVID-19 can be released from isolation. However, in cases of suspected COVID-19, physicians generally tend to do less thorough examinations. This is because several studies have shown that certain medical procedures carry a high risk of viral exposure for medical staff. ${ }^{26,27}$ Moreover, patients with suspected COVID-19 must be isolated from other patients owing to the risk of infection via aerosols that can remain in the air for several hours. ${ }^{28,29}$ Therefore, it is easy to fail to reduce risk or narrow the differential diagnosis and delay the start of therapy. A CT scan seems unnecessary for diagnosis in many cases, and the time lost before the start of therapy could be reduced. However, from the perspectives of protecting medical staff, conserving resources, and returning to the normal diagnostic flow, use of the CT-first triage protocol is acceptable.

We should emphasize that physicians are susceptible to cognitive bias (confirming bias) with respect to the clinical history and CT features of COVID-19. ${ }^{30,31}$ Some of our patients had life-threatening conditions that may mimic COVID-19. Because the symptoms and chest CT features of COVID-19 are non-specific, the CT-first triage protocol overestimates patients with COVID-19. ${ }^{15,32}$ Our results show that the CT-first 
triage protocol is useful for exclusive diagnosis of COVID-19. As mentioned, several life-threatening conditions that mimic COVID-19 were present in our outpatients. Therefore, medical doctors must be aware that patients suspected of COVID-19 may have any one of a number of diseases. Careful assessment of vital signs and medical interviews is important, and wide differential diagnosis and checking for life-threatening diseases are crucial.

There are some limitations in our study. First, this was a retrospective review at a single center. The characteristics of outpatients with suspected COVID-19 may depend on the study region and hospital characteristics. Our hospital is a tertiary-level hospital in Yokohama City and the patients in our hospital had many comorbidities; therefore, patient bias may be present. Furthermore, a hospital's medical protocol depends its medical resources; future validation studies are needed to confirm our results. Second, the number of patients was limited; therefore, further studies among outpatients with suspected COVID-19 are needed to yield more concrete evidence and confirm our findings. Third, in our study, we attempted to reduce exposure to radiation; however, the radiation dose did not reach the level of low-dose CT. Thus, radiation exposure is a potential limitation of this study. In accordance with the latest articles, we continue to revise our CT protocol to reduce the radiation dose to within a range suitable for triage. Finally, we did not perform SARS-CoV-2 PCR testing in the group of patients classified as less likely to have COVID-19. Thus, we might have missed COVID-19 cases in this group because many patients with COVID-19 have mild or no symptoms. ${ }^{1,33}$ However, no patients were diagnosed with COVID-19 in this group during follow-up, and there were no cases of secondary infection originating from COVID-19. Furthermore, one purpose of establishing a clinic for outpatients with suspected COVID-19 is to separate those suspected of having COVID-19 from other patients and to protect medical staff. In that sense, the CT-first triage protocol was useful; no medical staff infections or in-hospital infections occurred during the study period.

\title{
Conclusion
}

We demonstrated the efficacy and safety of the CT-first triage protocol for patients with suspected COVID19 in a real-world setting. Our CT-first triage protocol is acceptable for triaging patients with suspected COVID-19. Whereas the CT-first triage protocol is adequate for triage of COVID-19, there is a risk of delayed diagnosis and the start of therapy for life-threating conditions that mimic COVID-19. Our findings might improve the accuracy of diagnosis for outpatients with suspected COVID-19 in the present era of COVID-19 and can be helpful for appropriate triage, especially in settings where PCR testing is limited.

\section{Abbreviations}

\author{
Al: Artificial intelligence
}

CO-RADS: Coronavirus disease 2019 Reporting and Data System

COVID-19: Coronavirus disease 2019 
CT: Computed tomography

CTDIvol: CT dose index volume

GGO: Ground-glass opacities

PCR: Polymerase chain reaction

PPE: Personal protective equipment

SARS-CoV-2: Severe acute respiratory syndrome coronavirus 2

\section{Declarations}

\section{Acknowledgments}

We wish to thank all the staff at our institution for their support in COVID-19 treatment, as well as the study participants and their families. We thank Analisa Avila, ELS, and Emily Woodhouse, PhD, of Edanz Group (https://en-author-services.edanzgroup.com/ac) for editing a draft of this manuscript.

\section{Ethical approval and consent of participate}

The Institutional Review Board at Yokohama City University Hospital approved this study (approval number B200200047). Consent for participation in this study was obtained from all patients after explaining the clinical study by verbal or a description of how to opt out (https://www.yokohamacu.ac.jp/amedrc/ethics/ethical/fuzoku_optout.html).

\section{Consent for publication}

Not applicable

\section{Availability of data and materials}

The datasets used and/or analyzed during the current study will be available from the corresponding author on reasonable request.

\section{Competing interests}

None of the authors has any financial interests relevant to this trial to disclose.

\section{Funding and role of funder}

This research did not receive any specific grant from funding agencies in the public, commercial, or notfor-profit sectors.

\section{Author's contributions:}


SM, and TH conceived the study, designed the trial. TJ, TA, FO, YO, KT, YH, NK and HK undertook recruitment of participating centers and patients and managed the data, including quality control. TY and DU conducted radiological analyses. AN, TY, SM, TK and IT supervised the conduct of the trial and data collection. All authors contributed substantially to its revision. TH takes responsibility for the paper as a whole.

All authors attest to meeting the four ICMJE.org authorship criteria: (1) Substantial contributions to the conception or design of the work; or the acquisition, analysis, or interpretation of data for the work; AND (2) Drafting the work or revising it critically for important intellectual content; AND (3) Final approval of the version to be published; AND (4) Agreement to be accountable for all aspects of the work in ensuring that questions related to the accuracy or integrity of any part of the work are appropriately investigated and resolved.

\section{References}

1. Guan WJ, Ni ZY, Hu Y, et al. Clinical Characteristics of Coronavirus Disease 2019 in China. N Engl J Med. 2020;382(18):1708-1720.

2. Richardson S, Hirsch JS, Narasimhan M, et al. Presenting Characteristics, Comorbidities, and Outcomes Among 5700 Patients Hospitalized With COVID-19 in the New York City Area. JAMA. 2020.

3. Maffioli EM. How Is the World Responding to the 2019 Coronavirus Disease Compared with the 2014 West African Ebola Epidemic? The Importance of China as a Player in the Global Economy. Am J Trop Med Hyg. 2020.

4. Coronavirus disease (COVID-2019) situation reports. 2020;

https://www.who.int/emergencies/diseases/novel-coronavirus-2019/situation-reports. Accessed June 4, 2020.

5. Chen S, Zhang Z, Yang J, et al. Fangcang shelter hospitals: a novel concept for responding to public health emergencies. The Lancet. 2020;395(10232):1305-1314.

6. Bulut C, Kato Y. Epidemiology of COVID-19. Turk J Med Sci. 2020;50(SI-1):563-570.

7. Wu C, Chen X, Cai Y, et al. Risk Factors Associated With Acute Respiratory Distress Syndrome and Death in Patients With Coronavirus Disease 2019 Pneumonia in Wuhan, China. JAMA Intern Med. 2020.

8. Whiteside T, Kane E, Aljohani B, Alsamman M, Pourmand A. Redesigning emergency department operations amidst a viral pandemic. Am J Emerg Med. 2020.

9. Maves RC, Downar J, Dichter JR, et al. Triage of Scarce Critical Care Resources in COVID-19 An Implementation Guide for Regional Allocation: An Expert Panel Report of the Task Force for Mass Critical Care and the American College of Chest Physicians. Chest. 2020.

10. Wang Q, Wang X, Lin H. The role of triage in the prevention and control of COVID-19. Infect Control Hosp Epidemiol. 2020:1-15. 
11. Judson TJ, Odisho AY, Neinstein AB, et al. Rapid Design and Implementation of an Integrated Patient Self-Triage and Self-Scheduling Tool for COVID-19. J Am Med Inform Assoc. 2020.

12. Mossa-Basha M, Medverd J, Linnau K, et al. Policies and Guidelines for COVID-19 Preparedness: Experiences from the University of Washington. Radiology. 2020:201326.

13. Bernheim A, Mei X, Huang M, et al. Chest CT Findings in Coronavirus Disease-19 (COVID-19): Relationship to Duration of Infection. Radiology. 2020:200463.

14. Shi $\mathrm{H}, \mathrm{Han} X$, Jiang N, et al. Radiological findings from 81 patients with COVID-19 pneumonia in Wuhan, China: a descriptive study. The Lancet Infectious Diseases. 2020;20(4):425-434.

15. Rubin GD, Ryerson CJ, Haramati LB, et al. The Role of Chest Imaging in Patient Management during the COVID-19 Pandemic: A Multinational Consensus Statement from the Fleischner Society. Radiology. 2020:201365.

16. Coronavirus disease 2019 (COVID-19) situation within and outside the country. 2020; https://www.mhlw.go.jp/stf/seisakunitsuite/bunya/newpage_00032.html. Accessed May 19, 2020.

17. Takeuchi I. COVID-19 first stage in Japan - how we treat 'Diamond Princess Cruise Ship' with 3700 passengers? Acute Med Surg. 2020;7(1):e506.

18. Kato H, Shimizu H, Shibue Y, et al. Clinical course of 2019 novel coronavirus disease (COVID-19) in individuals present during the outbreak on the Diamond Princess cruise ship. $J$ Infect Chemother. 2020.

19. Ai T, Yang Z, Hou H, et al. Correlation of Chest CT and RT-PCR Testing in Coronavirus Disease 2019 (COVID-19) in China: A Report of 1014 Cases. Radiology. 2020:200642.

20. Long C, Xu H, Shen Q, et al. Diagnosis of the Coronavirus disease (COVID-19): rRT-PCR or CT? Eur J Radiol. 2020;126:108961.

21. Prokop $M$, van Everdingen W, van Rees Vellinga T, et al. CO-RADS - A categorical CT assessment scheme for patients with suspected COVID-19: definition and evaluation. Radiology. 2020:201473.

22. Li L, Qin L, Xu Z, et al. Artificial Intelligence Distinguishes COVID-19 from Community Acquired Pneumonia on Chest CT. Radiology. 2020:200905.

23. Xiang F, Wang X, He X, et al. Antibody Detection and Dynamic Characteristics in Patients with COVID19. Clin Infect Dis. 2020.

24. Li Z, Yi Y, Luo X, et al. Development and clinical application of a rapid IgM-IgG combined antibody test for SARS-CoV-2 infection diagnosis. J Med Virol. 2020.

25. Wang Z, Tang K. Combating COVID-19: health equity matters. Nat Med. 2020;26(4):458.

26. Chiu PWY, Ng SC, Inoue H, et al. Practice of endoscopy during COVID-19 pandemic: position statements of the Asian Pacific Society for Digestive Endoscopy (APSDE-COVID statements). Gut. 2020.

27. Lim LW, Yip LW, Tay HW, et al. Sustainable practice of ophthalmology during COVID-19: challenges and solutions. Graefes Arch Clin Exp Ophthalmol. 2020. 
28. Anderson EL, Turnham P, Griffın JR, Clarke CC. Consideration of the Aerosol Transmission for COVID19 and Public Health. Risk Anal. 2020.

29. Wang J, Du G. COVID-19 may transmit through aerosol. Ir J Med Sci. 2020.

30. Croskerry P. From mindless to mindful practice--cognitive bias and clinical decision making. N Engl J Med. 2013;368(26):2445-2448.

31. Talluri BC, Urai AE, Tsetsos K, Usher M, Donner TH. Confirmation Bias through Selective Overweighting of Choice-Consistent Evidence. Curr Biol. 2018;28(19):3128-3135 e3128.

32. Nasir MU, Roberts J, Muller NL, et al. The Role of Emergency Radiology in COVID-19: From Preparedness to Diagnosis. Can Assoc Radiol J. 2020:846537120916419.

33. Verity R, Okell LC, Dorigatti I, et al. Estimates of the severity of coronavirus disease 2019: a modelbased analysis. The Lancet Infectious Diseases. 2020.

\section{Tables}

Table 1. Characteristics of outpatients with suspected COVID-19

$$
\text { Characteristics } \quad \mathrm{N}=108
$$

Age, y (mean $\pm \mathrm{SD}$, range) $58.9 \pm 19.5$ range: $18-101$

Sex

\begin{tabular}{|c|c|c|}
\hline Male & 60 & $55.6 \%$ \\
\hline Female & 48 & $44.4 \%$ \\
\hline \multicolumn{3}{|l|}{ Clinical history } \\
\hline Overseas travel history & 4 & $3.7 \%$ \\
\hline Contact with a COVID-19 case & 8 & $7.4 \%$ \\
\hline \multicolumn{3}{|l|}{ Comorbidity } \\
\hline Any & 74 & $68.5 \%$ \\
\hline Chronic pulmonary disease & 21 & $19.4 \%$ \\
\hline Diabetes & 14 & $13.0 \%$ \\
\hline Hypertension & 25 & $23.1 \%$ \\
\hline Chronic renal failure & 6 & $5.6 \%$ \\
\hline Cardiovascular disease & 10 & $9.3 \%$ \\
\hline Cerebrovascular disease & 4 & $3.7 \%$ \\
\hline Malignancy & 20 & $18.5 \%$ \\
\hline \multicolumn{3}{|l|}{ Symptoms } \\
\hline Fever & 77 & $71.2 \%$ \\
\hline Respiratory symptoms & 55 & $50.9 \%$ \\
\hline Fatigue & 37 & $34.3 \%$ \\
\hline Headache & 20 & $18.5 \%$ \\
\hline Taste or olfactory disorder & 7 & $6.5 \%$ \\
\hline
\end{tabular}

Abbreviations: COVID-19, Coronavirus disease 2019. 
Note: Fever, body temperature above $37.5^{\circ} \mathrm{C}$.

Table 2. Results of CT-first protocol

COVID-19 suspected ( $\mathrm{n}$ COVID-19 less likely ( $\mathrm{n}$

Patients

$48=48$ )

$44.9 \%$
$=59$ )

Age, y (mean $\pm \mathrm{SD}$, range)

Sex (number, \%)

$61.1 \pm$
17.8

27

range: $21-$

90

$56.3 \%$

21

$43.8 \%$

59

$55.1 \%$

\section{Female}

21

26
$57.3 \pm$
20.9

range: $18-$ 101

\section{CT feature}

CO-RADS
(number, \%)

Category 1
Category 2
Category 3

6

$12.5 \%$

$18.8 \%$

42

$29.2 \%$

17

$55.9 \%$

Category 4

14

$12.5 \%$

0

$44.1 \%$

\section{Category 5}

13

$27.1 \%$

$81.7 \pm$ 48.8 $1013 \pm$ $\begin{array}{cc}505.5 & 1665 \\ 1037.0 \pm & \text { range: } 255\end{array}$

$$
\text { range) }
$$

$($ mean $\pm \mathrm{SD}$

Time until PCR confirmatio range)

Isolation duration, non-COVID-19 group, min (mean $\pm \mathrm{SD}$, range)

$1037.0 \pm$ range: 255 $512 \quad 1665$

range: $23-\quad 70.5 \pm 41.2$ range: 19 $289 \quad 297$ range: $255-$

$71.2 \%$
$28.8 \%$
$0.0 \%$
$0.0 \%$
$0.0 \%$

$0.0 \%$

Abbreviations: COVID-19, Coronavirus disease 2019, CO-RADS, Coronavirus disease 2019 Reporting and Data System, CT, Computed tomography, PCR, Polymerase chain reaction.

Table 3. Clinical outcomes 
COVID-19 suspected $(\mathrm{n}=48$ ) COVID-19 less likely $(\mathrm{n}=59)$

\begin{tabular}{ccccc}
\hline Final diagnosis & & & & \\
COVID-19 & 9 & $18.8 \%$ & 0 & $0.0 \%$ \\
\hline Not COVID-19 & 39 & $81.2 \%$ & 59 & $100 \%$ \\
\hline Respiratory infection & 23 & & & \\
\hline Other focal infection (meningitis) & $7(2)$ & $14.6 \%(4.2 \%)$ & $15(2)$ & $25.4 \%(3.4 \%)$ \\
\hline Interstitial pneumoniae & 4 & $8.3 \%$ & 0 & $0.0 \%$ \\
\hline Tumor related fever & 2 & $4.2 \%$ & 3 & $5.1 \%$ \\
\hline Collagen disease & 0 & $0.0 \%$ & 2 & $3.4 \%$ \\
\hline Acute myocardial infarction & 0 & $0.0 \%$ & 1 & $1.7 \%$ \\
\hline Others & 3 & $6.3 \%$ & 10 & $16.9 \%$ \\
\hline Clinical outcome & & & & \\
\hline Death & 1 & $2.1 \%$ & 1 & $1.7 \%$ \\
\hline Hospitalization & 31 & $64.6 \%$ & 20 & $33.9 \%$ \\
\hline Not hospitalized & 17 & $35.4 \%$ & 39 & $66.1 \%$ \\
\hline
\end{tabular}

Abbreviations: COVID-19, Coronavirus disease 2019.

\section{Figures}


A
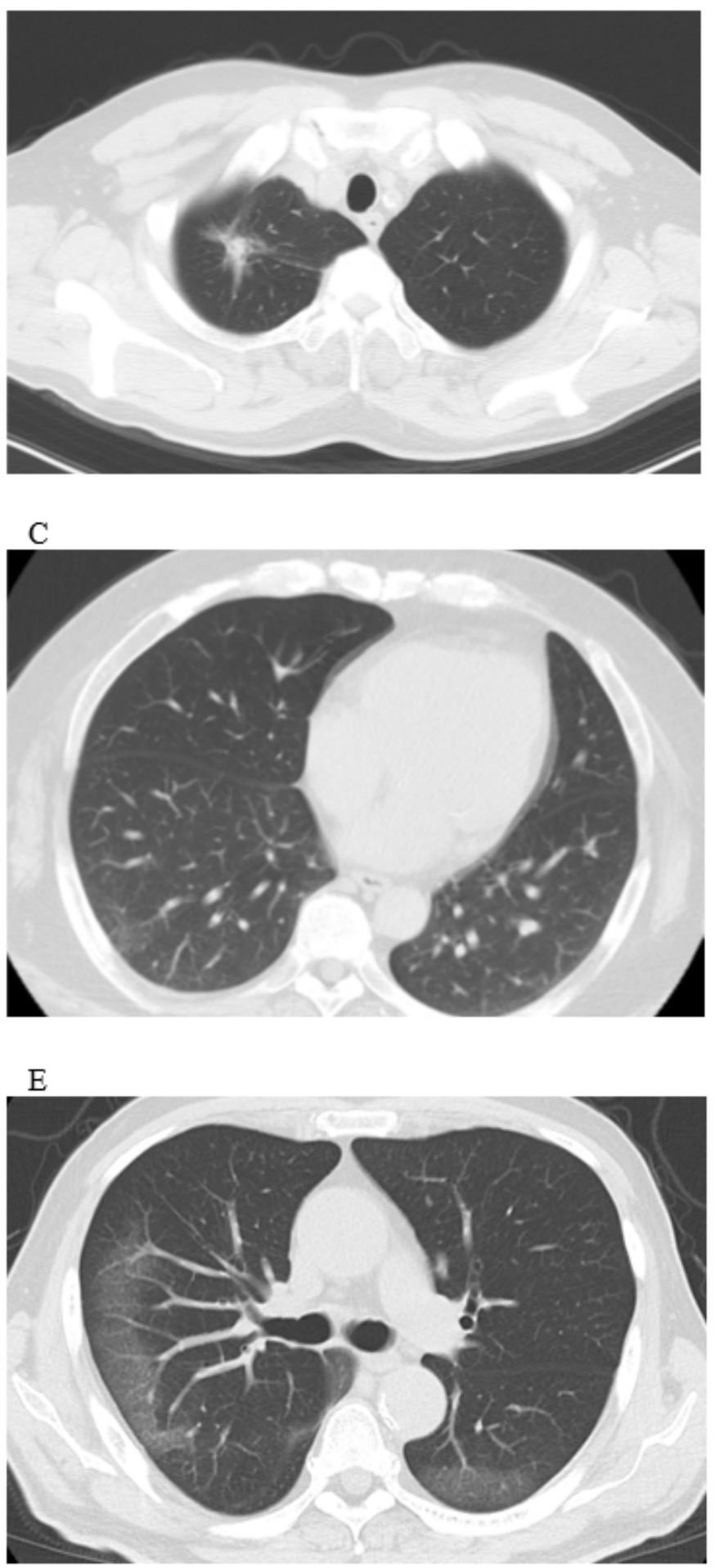

B

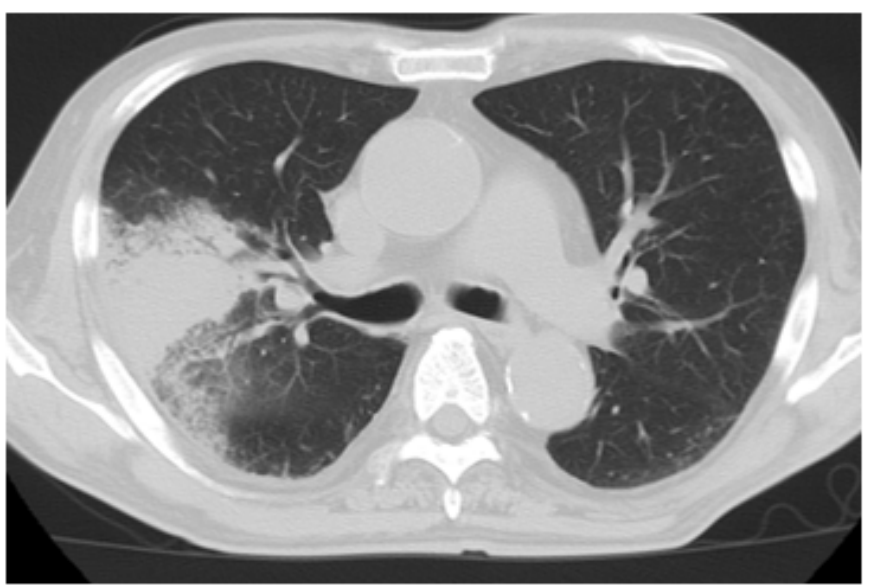

$\mathrm{D}$

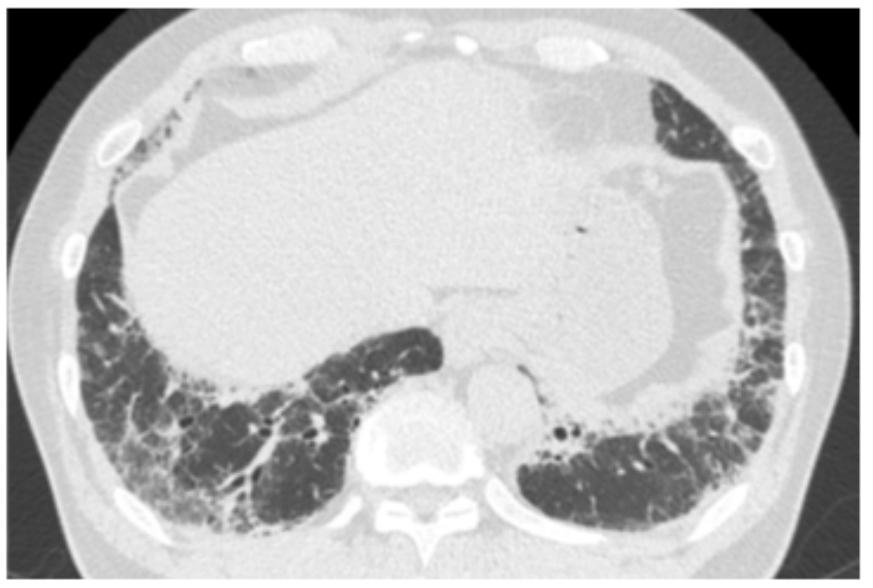

\section{Figure 1}

Representative features of chest computed tomography (CT) classifications Chest CT scans of outpatients with suspected COVID-19. CT features classified into five categories according to the COVID19 Reporting and Data System (CO-RADS). A) CO-RADS 1, normal CT or non-infectious etiology (e.g., lung tumor, lung fibrosis). B) CO-RADS 2, infectious etiology that is not compatible with COVID-19 (e.g., infectious bronchiolitis, bronchopneumonia, lobar pneumonia). C) CO-RADS 3, equivocal findings for 
COVID-19 (e.g., homogeneous extensive ground-glass opacities (arrow)). D) CO-RADS 4, typical CT findings of COVID-19 (similar to CO-RADS 5) but showing some overlap with other pneumonia. E) CORADS 5, typical CT findings of COVID-19 (e.g., multifocal ground-glass opacities with or without consolidation in lung regions close to visceral pleural surfaces)

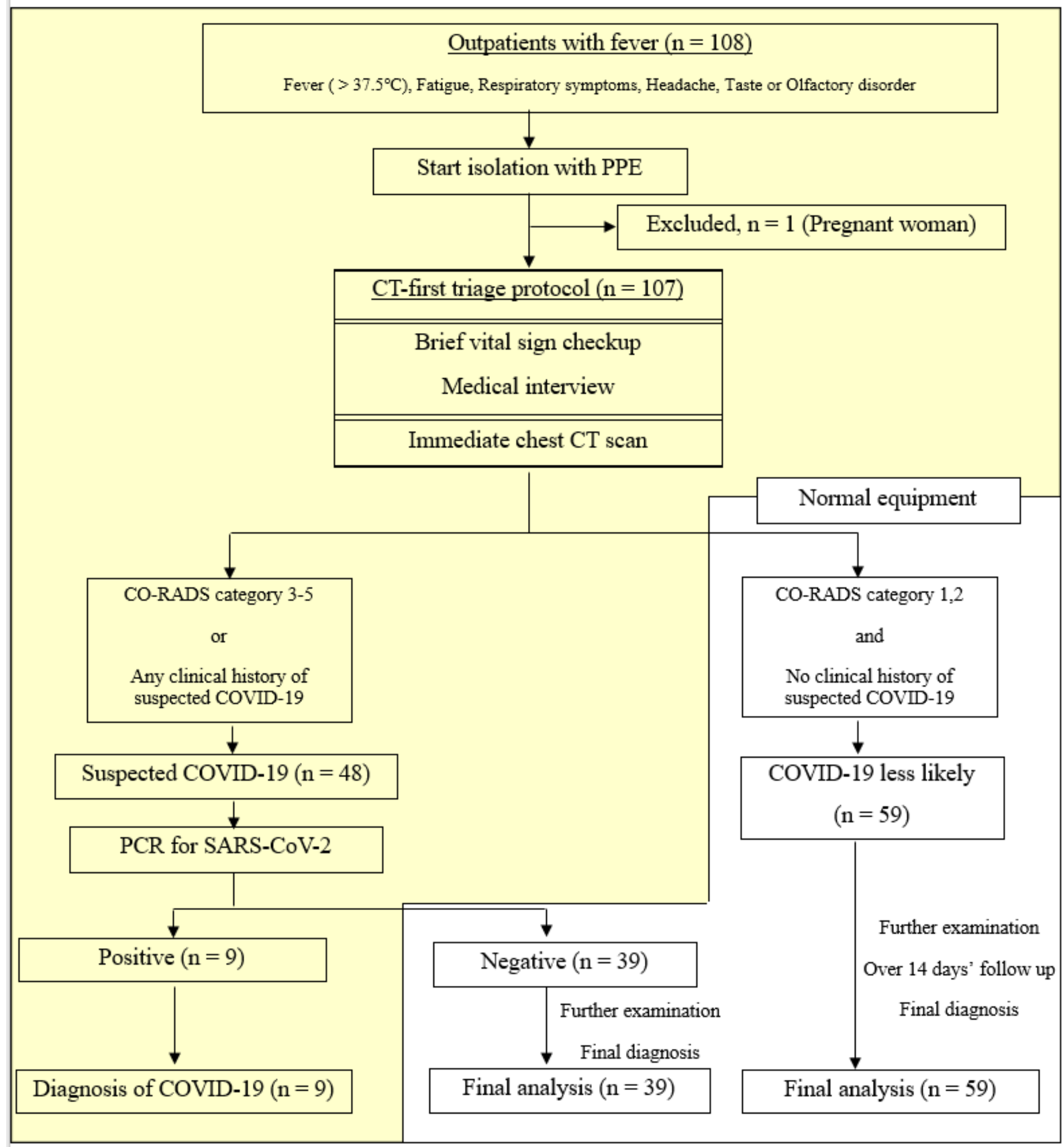

Figure 2 
Computed tomography (CT)-first triage protocol for outpatients with suspected COVID-19 and participant flow. Overview of the CT-first triage protocol for outpatients with suspected COVID-19 and participant flow. Yellow area indicates duration of isolation, requiring personal protective equipment (PPE). One patient was excluded from the CT-first triage protocol because of pregnancy; the remainder were triaged using the protocol. Patients in the group less likely to have Coronavirus disease 2019 (COVID-19), classified according to the COVID-19 Reporting and Data System (CO-RADS) and the patient's clinical history, were treated using normal equipment and were finally diagnosed after further examination. The "COVID-19 less likely" group was followed for 14 days after visiting our outpatient clinic. The COVID-19 suspected group was tested using polymerase chain reaction (PCR) for severe acute respiratory syndrome coronavirus 2 (SARS-CoV-2). Patients with negative PCR test results for SARS-Co-2 were treated using normal equipment and were finally diagnosed after further examination.

\section{Supplementary Files}

This is a list of supplementary files associated with this preprint. Click to download.

- Supplementalmaterial0612.docx 